\title{
The Coming-of-Age of Software Architecture Research
}

\section{The \\ Coming-of-Age \\ of \\ Software Architecture \\ Research}

\author{
Mary Shaw
}

Carnegie Mellon University

www.cs.cmu.edu/ shaw/

\section{Executive Summary}

- Technical ideas in software engineering evolve in predictable ways

- This evolution pattern sets expectations for software architecture research

- Lessons for developers: what to expect of software architecture research, now and in the future

- Lessons for researchers: useful research paradigms and validation techniques 


\section{The Coming-of-Age of Software Architecture Research}

\section{Engineering}

Definitions abound. They have in common:

Creating cost-effective solutions ...

... to practical problems ...

... by (preferring to) apply scientific knowledge ...

... building things ...

$\ldots$ in the service of mankind

Engineering entails making decisions under constraints of limited time, knowledge, and resources

SE research is a prime source of the systematic knowledge

SE research problems should reflect the constraints

\section{Software Architecture Research}

Software architecture research is the principled study of the overall structure of software systems, especially the relations among subsystems and components. 


\section{The Coming-of-Age of Software Architecture Research}

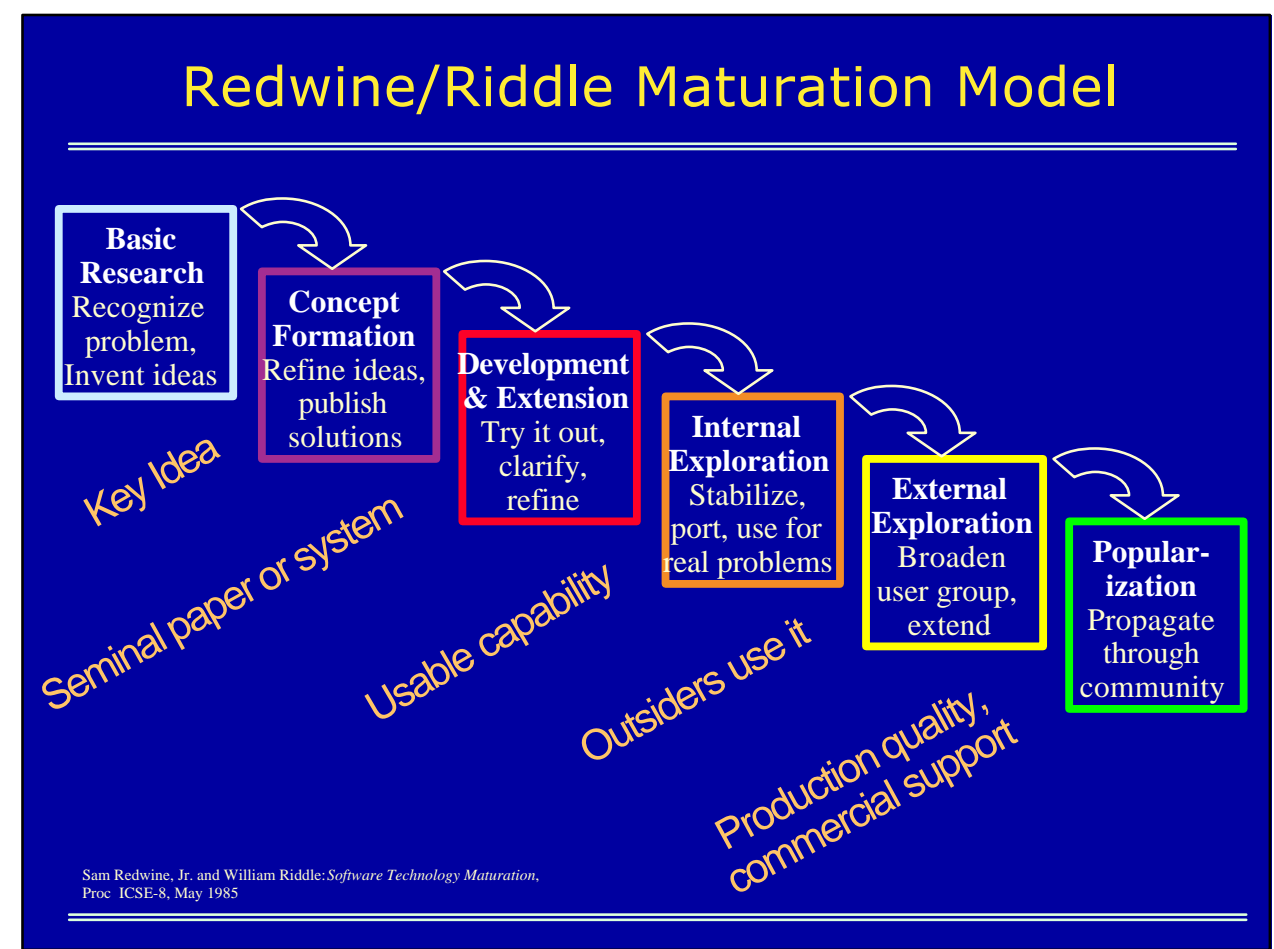

\section{Software Technology Maturation Points}

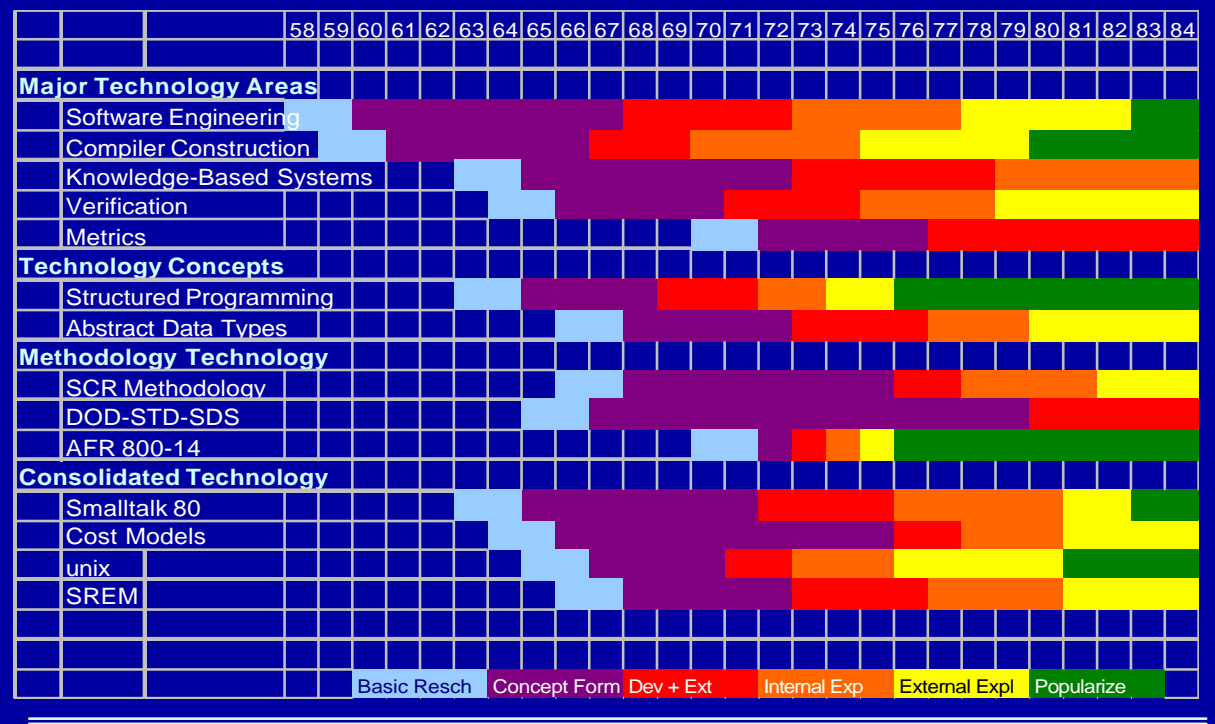




\section{The Coming-of-Age of Software Architecture Research}

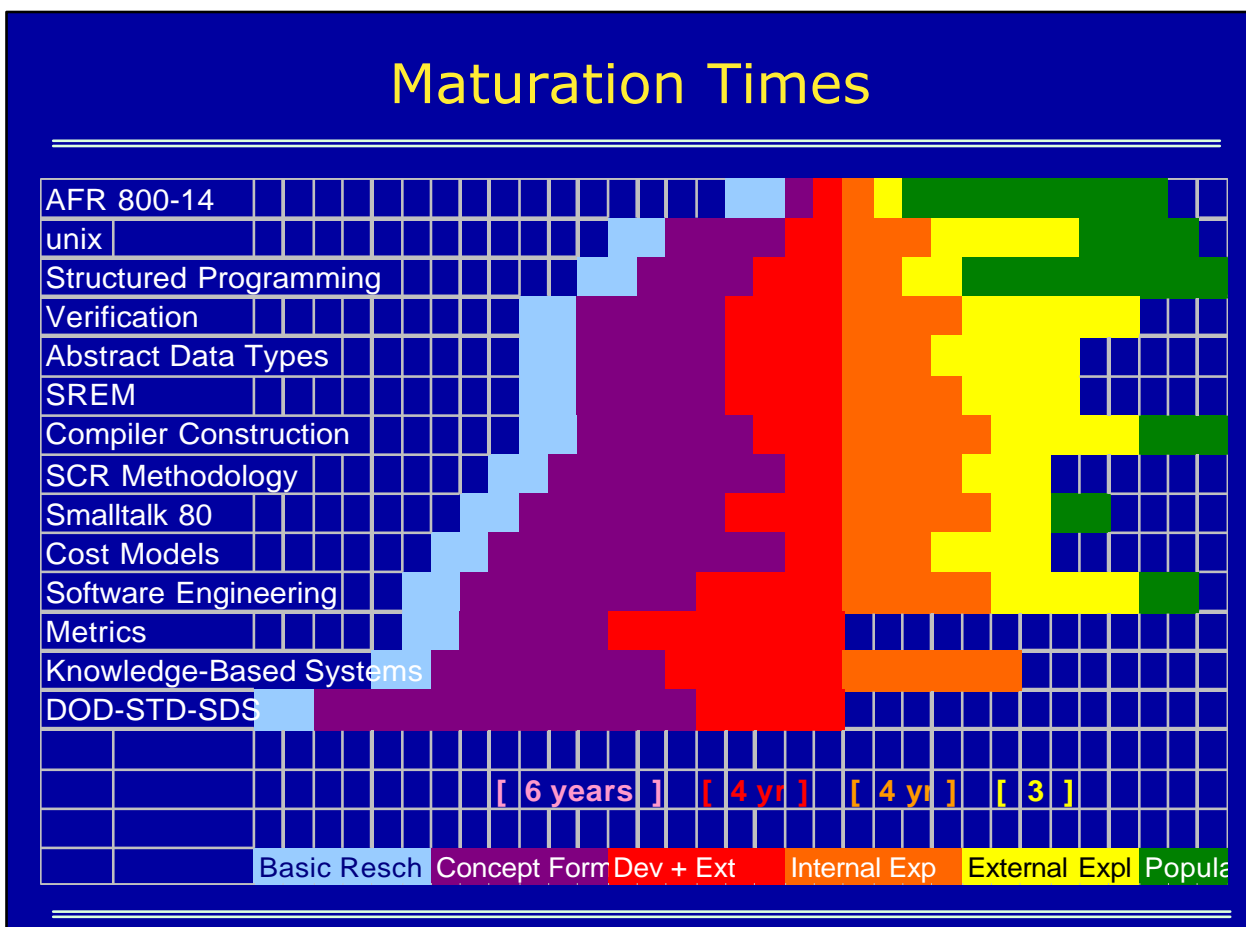

\section{Transition Points for Abstract Data Types}

- Basic research ==> concept formation

$>$ 1968: formulation of information hiding

- Concept formation ==> development \& extension

$>$ 1973: abstract data type models

- Development \& extension ==> internal exploration

$>$ 1977: incorporation in programming languages

- Internal exploration $==>$ external exploration

$>$ 1980: incorporation in other technologies

- External exploration ==> Popularization

$>$ late 80's:object models,C+_,Java 


\section{The Coming-of-Age of Software Architecture Research}

\section{Basic Research in Software Architecture}

- Architectures for particular product lines or application domains (e.g., oscilloscopes, missile control)

- Catalogs that captured informal use of box and line diagrams

- Key idea: models for architectural style

Late 1980s to $1992 / 3$

\section{Concept Formulation in Software Architecture}

- Architecture description languages

> capture design abstractions in system definition

$>$ exploit properties of particular styles

> support style-specific compilation or analysis

- Prototype implementations support further experiments

- Formalization, both within language and standalone

- Catalogs expanded to taxonomies

Mid 1990s and ongoing 


\section{The Coming-of-Age of Software Architecture Research}

\section{"Software Architecture" citation counts}

\section{NEC Bibliography citation counts}

"software architecture" in entry (2000 of 3595 citations)

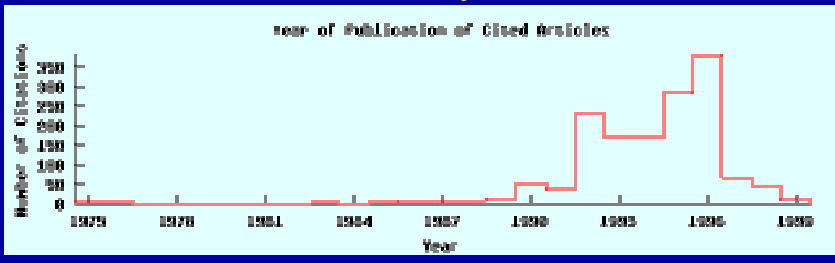

"software architecture" in title (1831 citations)

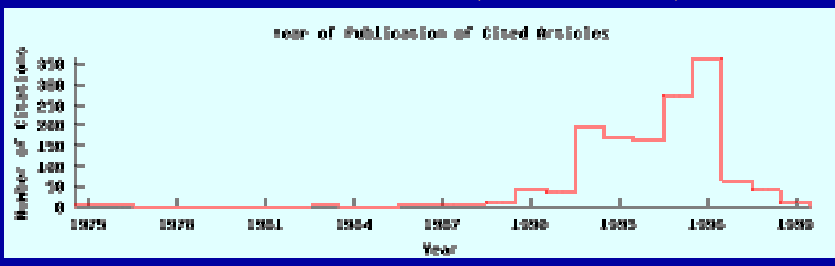

Falloff in 1997-99 may reflect delay in updating bibliography and/or normal publication lags

\section{Software Architecture 24 Most Cited}

- Published 1990-1998

- 4 books (1995-1998)

- 20 papers

$>8$ architectures for particular domains (1990-1995)

$>7$ surveys or models for field (1992-1995)

$>3$ formalizations (1993-1996)

$>1$ architecture description language (1995)

$>1$ reverse engineering (1997) 


\section{The Coming-of-Age of Software Architecture Research}

\section{Development and Extension in Software Architecture}

- Unification and refinement

$>$ ACME interchange language

$>$ refinement of taxonomies

- Maturing institutions

$>$ workshops, special issues, conference tracks

$>$ books

$>$ independent conference

Late 1990s and ongoing

\section{Internal Enhancement and Exploration} in Software Architecture

- Architectural styles used informally as design guides

- Some formal analyses of real systems

- Books/courses on software architecture

From 1998 onward 


\section{The Coming-of-Age of Software Architecture Research}

\section{External Enhancement and Exploration in Software Architecture}

- Recognition of the value of architects and architectural design considerations

$>$ Processes require architectural design reviews and explicit architectural documentation

$>$ Emerging use of product line architectures, commercial architectural standards, component integration frameworks

- Tool-supported methods

$>$ Architectural views in UML

$>$ Architectural Tradeoff Analysis Method

Very late 1990s and ongoing

\section{Popularization of Software Architecture}

- Emerging standards

> IEEE-Std-1471-2000, recommended Practice for Architectural Description of Software-Intensive Systems

> Standards for particular component families

» but these reflect component reuse interests as much as architecture

\section{Just beginning}




\section{The Coming-of-Age of Software Architecture Research}

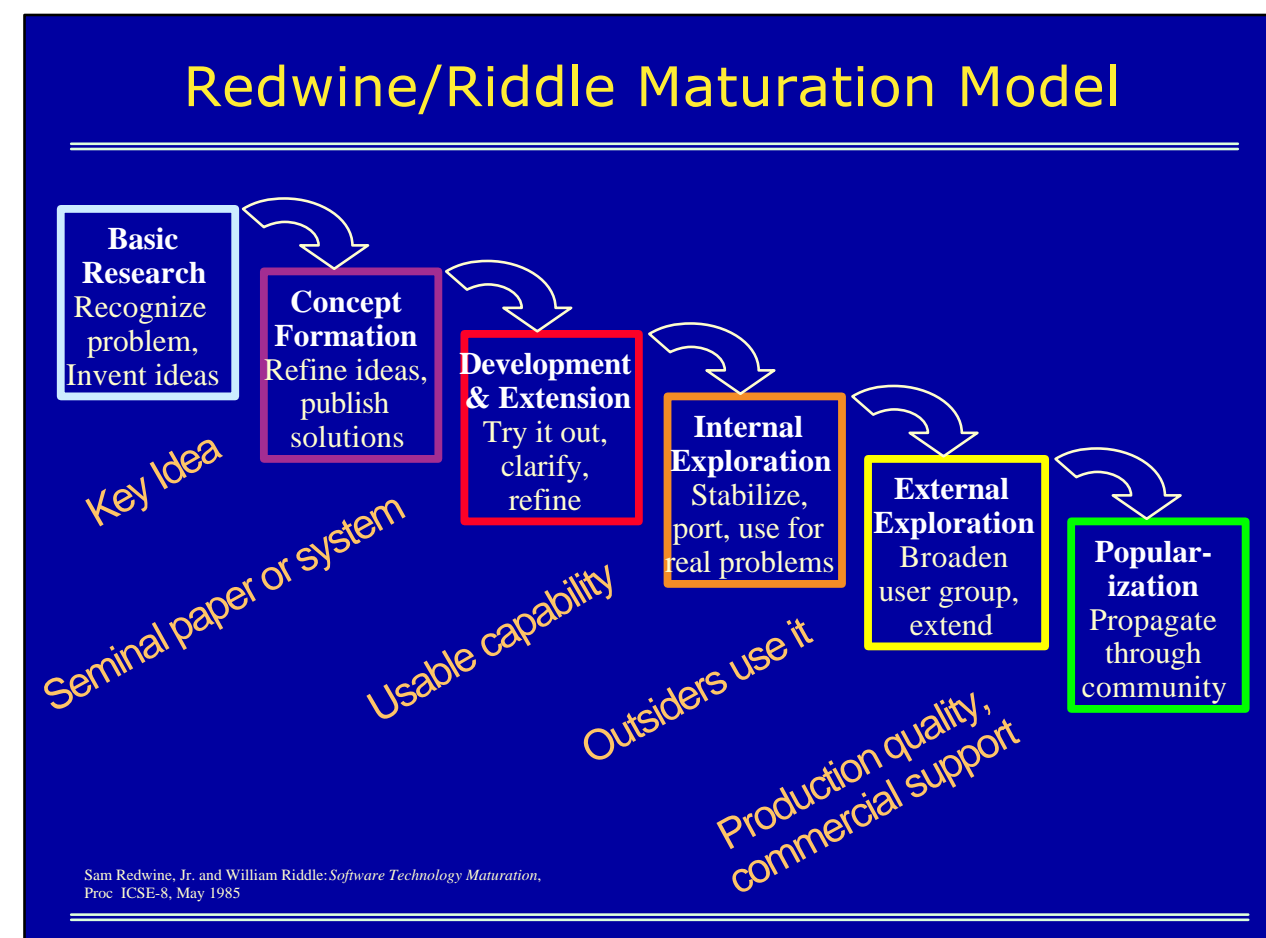

\section{Focus on Research}

- Recall ...

SE research is a prime source of the systematic knowledge SE research problems should reflect the constraints

- Turn now to research strategies 


\section{The Coming-of-Age of Software Architecture Research}

\section{Research Strategy}

Examine the kinds of research questions software engineers ask and the ways they study those questions

- Ideas mature over time

They grow from qualitative and empirical understanding to precise and quantitative models

- Research questions are of different kinds

Kinds of interesting questions change as ideas mature

- Research strategies also vary

They should be selected to match the research questions

\section{Research Objectives}

\section{Real World}

Practical problem

\section{Real World}

Solution to

nractical nrohlem

- Key objectives

$>$ Quality -- utility as well as functional correctness

$>$ Cost -- both of development and of use

> Timeliness -- good-enough result, when it's needed

- Address problems that affect practical software 


\section{The Coming-of-Age of Software Architecture Research}

\section{Types of Research Questions}

- Feasibility

Does $\mathrm{X}$ exist, and what is it?

Is it possible to do $\mathrm{X}$ at all ?

- Characterization What are the characteristics of X ?

What, exactly, do we mean by $\mathrm{X}$ ?

What are the varieties of $\mathrm{X}$, and how are they related?

- Method/Means How can we do X ?

What is a better way to do $\mathrm{X}$ ?

How can we automate doing $\mathrm{X}$ ?

- Generalization Is $\mathrm{X}$ always true of $\mathrm{Y}$ ?

Given $\mathrm{X}$, what will $\mathrm{Y}$ be ?

- Discrimination How do I decide whether X or Y ?

\section{Examples of Research Questions about} Software Structure

- Feasibility Is it possible to describe relations among components of a software system?

- Characterization What is the important information to share and hide about a component?

- Method/Means How can we incorporate abstract data types in a programming language?

- Generalization What is a formal relation between the specification of an abstract data type and its implementation?

- Discrimination What organization should I choose for a user interface component? 


\section{The Coming-of-Age of Software Architecture Research}
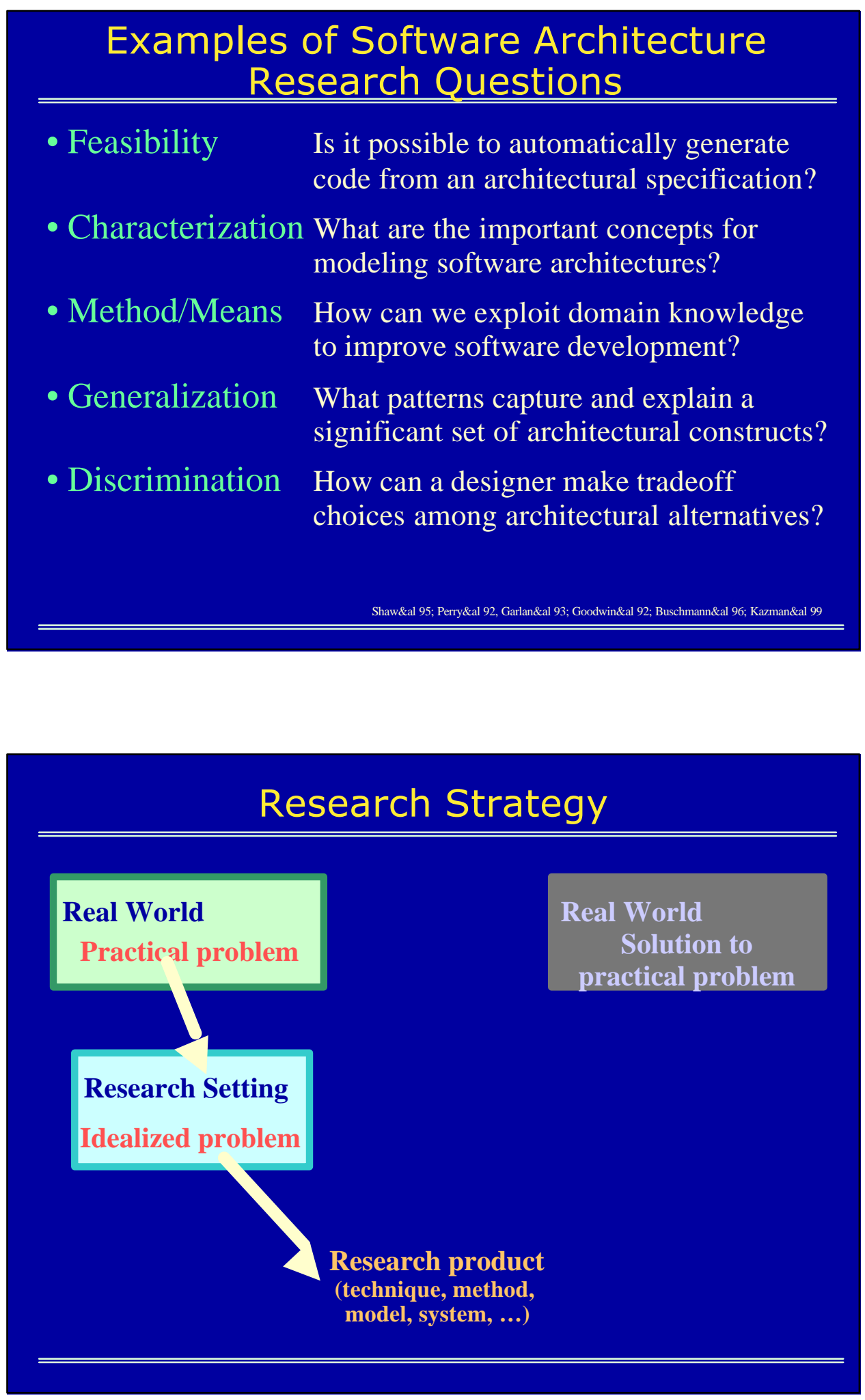


\section{The Coming-of-Age of Software Architecture Research}

\section{Types of Research Results}

- Qualitative \& Report interesting observations descriptive Generalize from (real-life) examples models Structure a problem area; ask good questions

- Techniques Invent new ways to do some tasks, including implementation techniques Develop ways to select from alternatives

- System Embody result in a system, using the system both for insight and as carrier of results

- Empirical Develop empirical predictive models from models observed data

- Analytic Develop structural models that permit models formal analysis

\section{Maturity: Kinds of Research Results}

Brooks proposed recognizing three kinds of results, with individual criteria for quality:

> findings -- well-established scientific truths -judged by truthfulness and rigor

> observations -- reports on actual phenomena -judged by interestingness

$>$ rules-of-thumb -- generalizations, signed by an author (but perhaps not fully supported by data) -judged by usefulness

with freshness as criterion for all 


\section{The Coming-of-Age of Software Architecture Research}

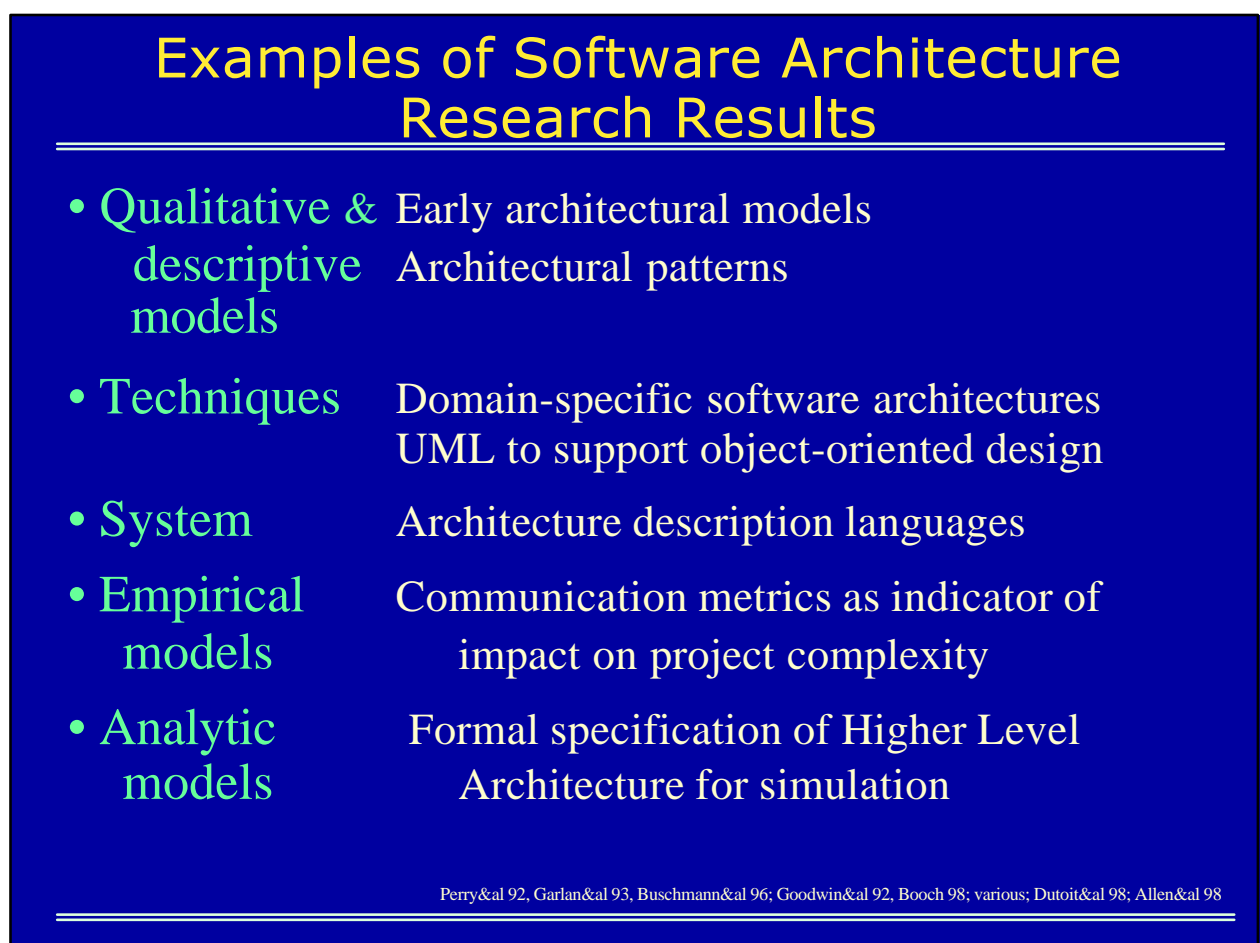

\section{Research Objectives}

\section{Real World}

Practical problem

Practical problen

\section{Real World}

Solution to

practical problem

\section{Research Setting}

Solution to

idealized problem

Does the product solve

the idealized problem?

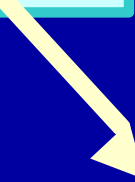

Research produc

(technique, method,

model, system, ...)

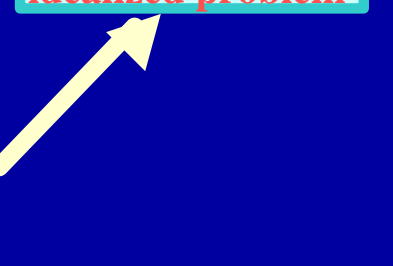




\section{The Coming-of-Age of Software Architecture Research}

\section{Types of Research Validation}

- Persuasion I thought hard about this, and I believe...

- Implementation Here is a prototype of a system that ...

- Evaluation Given these criteria, the object rates as ...

- Analysis Given the facts, here are consequences ...

Formal model Rigorous derivation and proof

Empirical model Data on use in controlled situation

- Experience Report on use in practice

Qualitative model Narrative

Decision criteria Comparison of systems in actual use

Empirical model Data, usually statistical, on practice

\section{Examples of Software Architecture} Research Validation

- Persuasion Early architectural models

- Implementation Early architecture description languages

- Evaluation Taxonomies; performance improvement

- Analysis (Many predictive models)

Formal model HLA for distributed simulation

Empirical model User interface structure

- Experience (Many case studies)

Qualitative model Architectural patterns

Comparison Domain-specific architectures

Empirical model Communication and project complexity 


\section{The Coming-of-Age of Software Architecture Research}

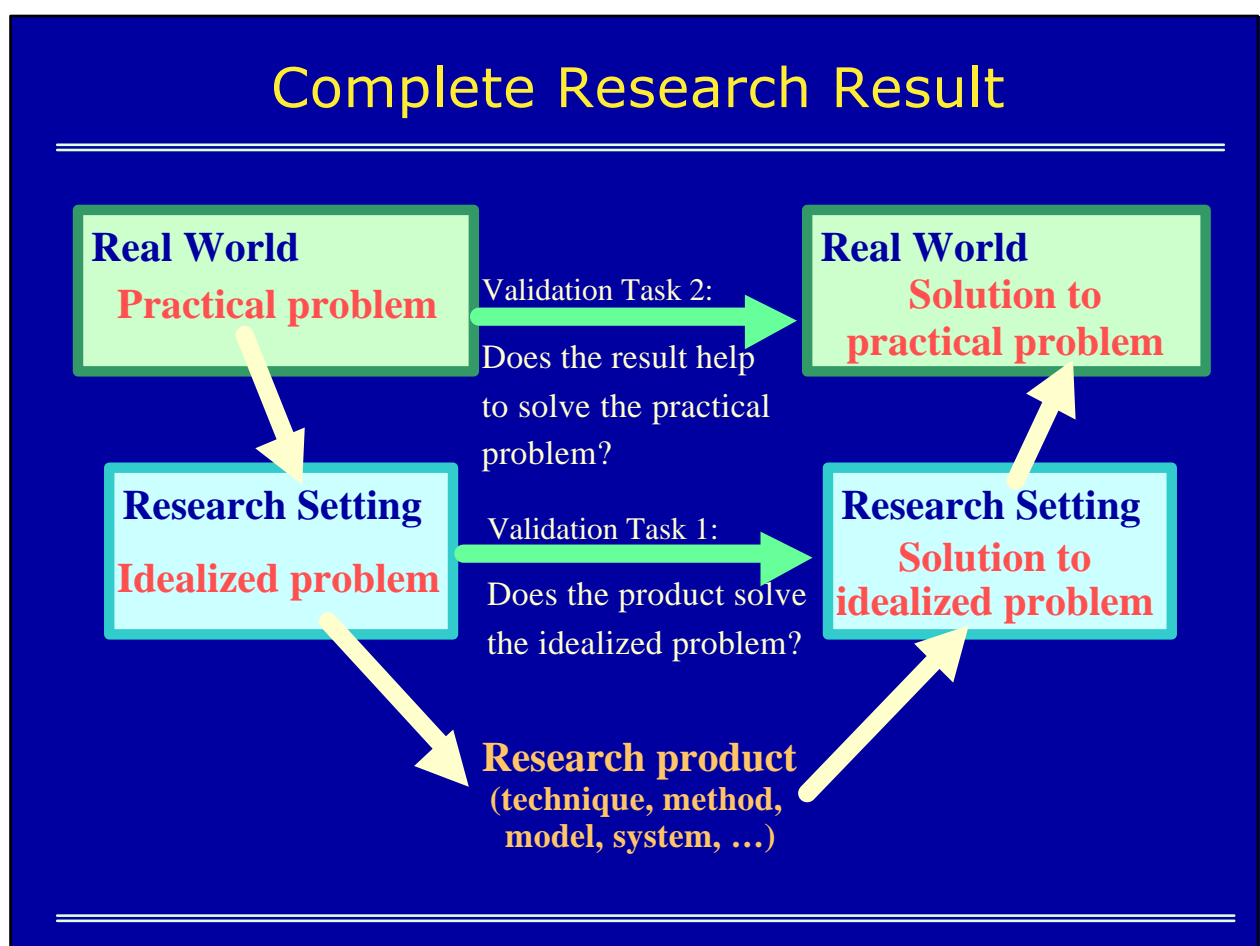

\section{"No-No" s for Software Engineering Research}

- Assume that a result demonstrated for a $10 \mathrm{~K}$-line system will scale to a $500 \mathrm{~K}$-line system

- Expect everyone to do things "my way"

- Believe functional correctness is sufficient

- Assume the existence of a complete, consistent specification

- Just build things without extracting enduring lessons

- Devise a solution in ignorance of how the world really works 


\section{The Coming-of-Age of Software Architecture Research}

\section{Building Blocks for Research}
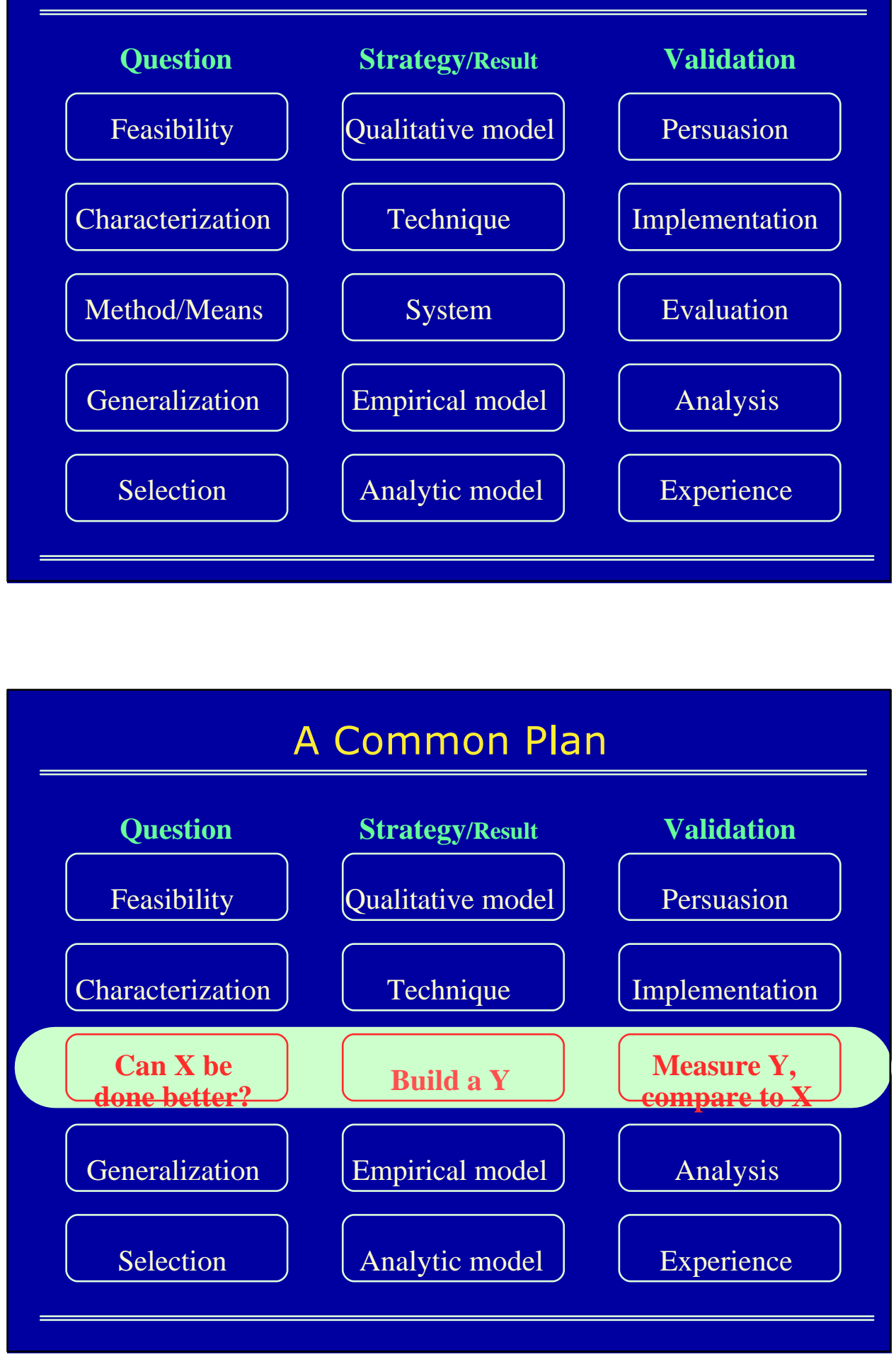


\section{The Coming-of-Age of Software Architecture Research}
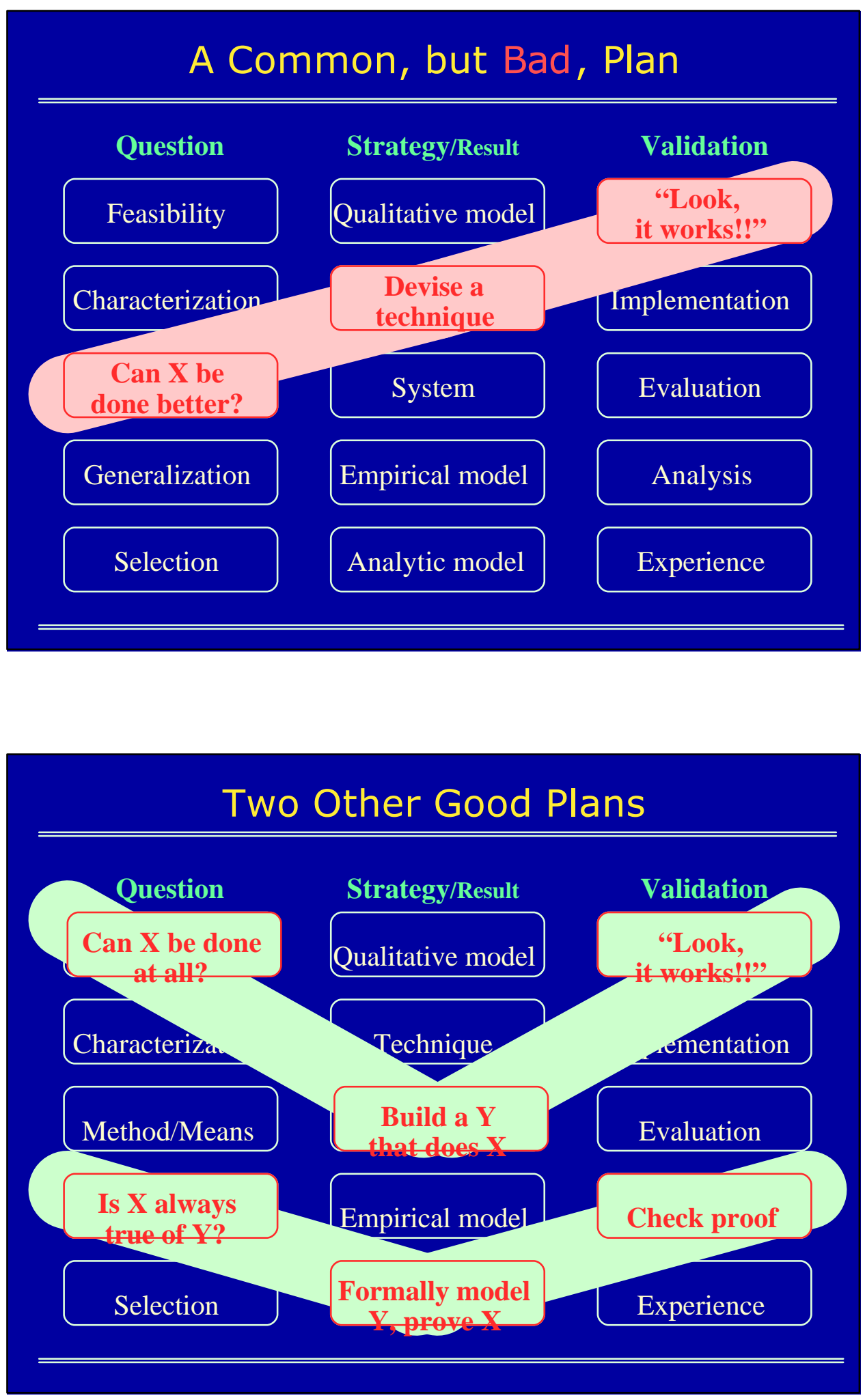


\section{The Coming-of-Age of Software Architecture Research}

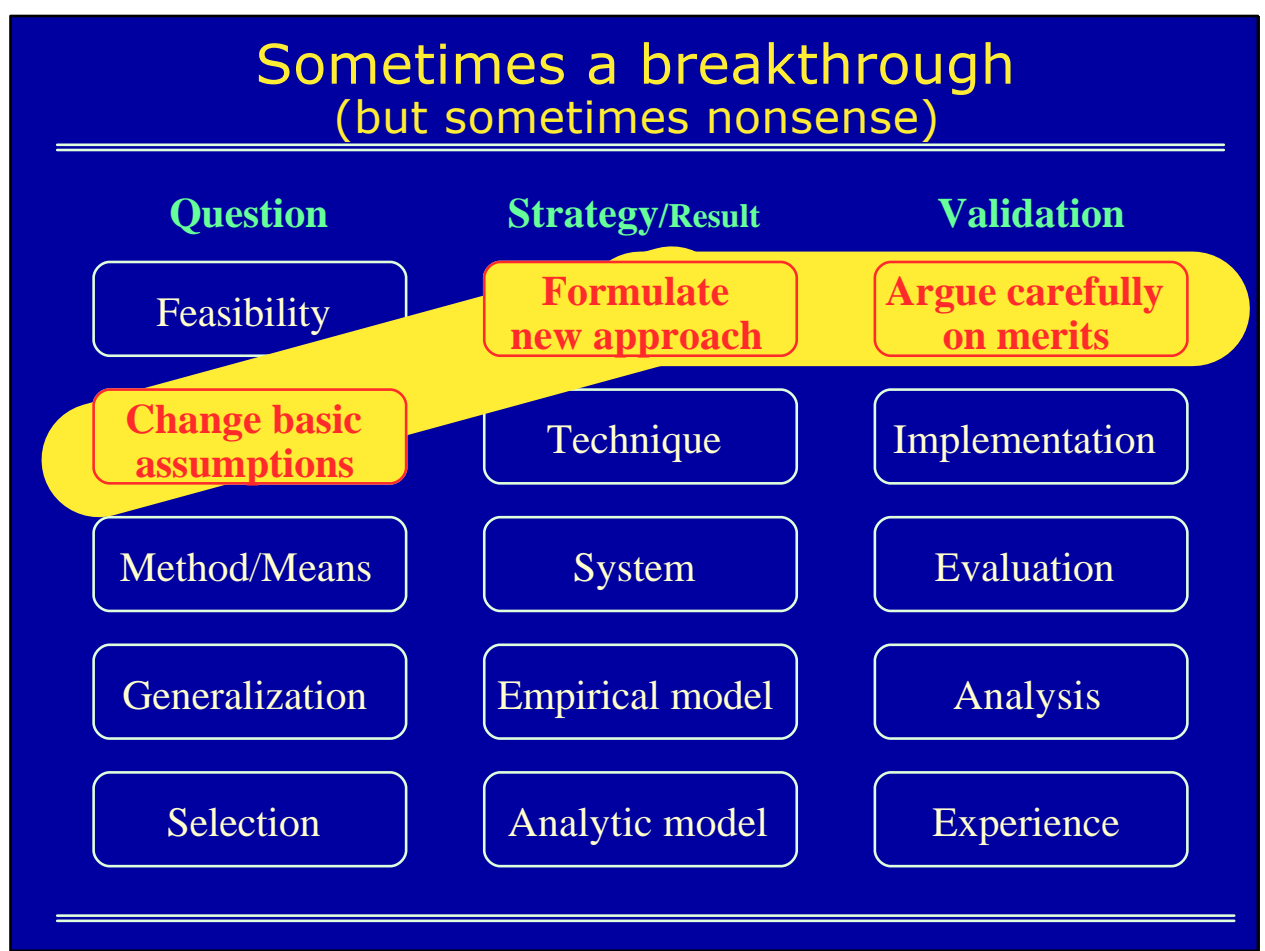

\section{Problem Types, Products, Validations}

24 classic SE papers, 10 ICSE 2001 software architecture papers

Qual / Tech- Syste Empir Analy

desc nique $\mathrm{m}$ ical tic

model model model

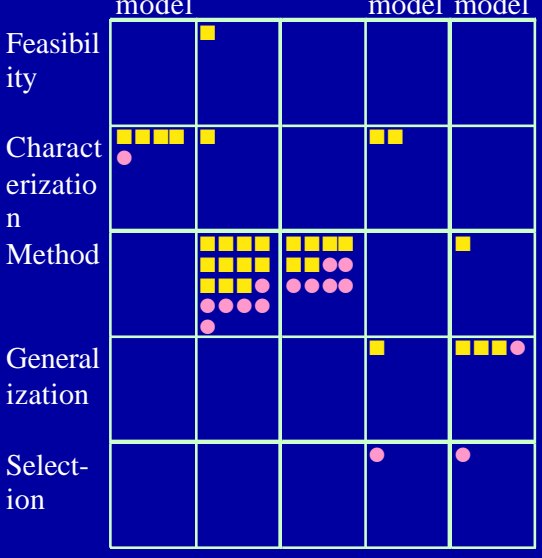

Persu Imple Evalu Analy Exper asion menta ation sis ience

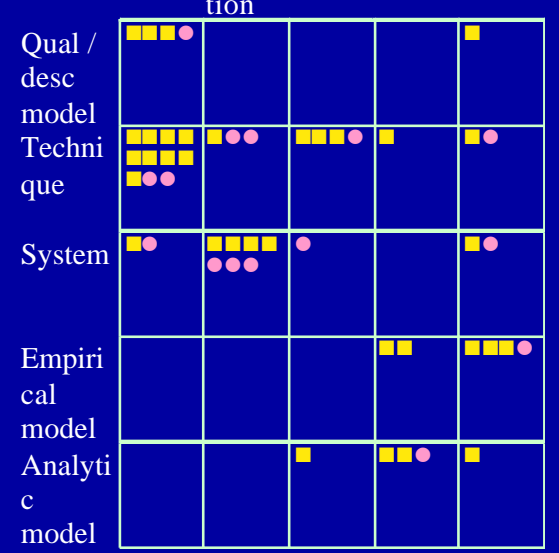




\section{The Coming-of-Age of Software Architecture Research}

\section{Executive Summary}

- Technical ideas in software engineering evolve in predictable ways

- This evolution pattern sets expectations for software architecture research

- Lessons for developers: what to expect of software architecture research, now and in the future

- Lessons for researchers: useful research paradigms and validation techniques

> I offer these as observations, in Brooks' sense

\section{What Next?}

- Software architecture

$>$ Don't just continue in the style of the 90's

$>$ Make existing results more robust, better evaluated

> Address new problems: highly distributed systems, dependability, humane systems

$>$ Help transition to practice

- Software engineering

$>$ Be more explicit about research paradigms, when and why they work

$>$ Be more careful about validation, especially real-world significance 